EGU2020-21592

https://doi.org/10.5194/egusphere-egu2020-21592

EGU General Assembly 2020

(c) Author(s) 2020. This work is distributed under

the Creative Commons Attribution 4.0 License.

\title{
Scour-resilient bio-inspired geomorphic designs: The Male Japanese Puffer Fish Nest
}

\author{
Laurence Clement, Callum Campbell, Arezoo Hasibi, Hossein Zare-Behtash, and Manousos \\ Valyrakis \\ University of Glasgow, School of Engineering, United Kingdom of Great Britain and Northern Ireland \\ (manosva21@gmail.com)
}

In 1995, divers noticed a strange circular pattern on the seabed off Japan. The geometric formations mysteriously appeared and dissolved, and no-one knew what made them. Finally, the creator of these remarkable formations was found: a new species of pufferfish from the genus Torquigener. The male puffer fish executes a design of mathematical perfection in the form of ornate circles. As he swims along the seabed, he laboriously flaps his fins and rearranges the sand, creating the geomorphic feature dubbed crop circle by the pioneers who first noticed them. The significance to understand the puffer fish design is magnified when we consider that the nest is able to maintain its morphological features for long periods even though it is built entirely of mobile particles in an area where the flow does not stop.

As a relatively new discovery, the exact reasons behind why the pufferfish spends such a long time constructing and cultivating the nest it still a question that is shrouded in a substantial amount of mystery. Male puffer fish spend many days caring for the eggs, the only puffer fish genus to be overserved doing so; suggesting that Torquigener place an unusually large emphasis on ensuring the survival of their eggs. It is hypothesised that the nest is created as a mating display, as female puffer fish will visit the site, presumably assessing various characteristics of the nest. It is not known exactly what parameters the females judge the nest on; whether it be size, symmetric properties or decorative choice. However, due to some basic hydrodynamic experiments performed by Hiroshi Kawase, there is some evidence to suggest that there may be more to building the nest than solely attracting a mate.

Several questions therefore arise regarding the nest. Is there an evolutionary reason that male puffer fish build these nests? Which characteristics of a nest make it attractive to female puffer fish? Are the eggs safer in a nest, perhaps from incoming currents? How exactly does fluid flow through nest, and can it be replicated and simulated? This project begins to tackle these questions through a numerical investigation (CFD) of the fluid flow through the nest in order to identify key fluid dynamic features, which may play a significant role in egg incubation and spawning using Star $\mathrm{CCM}+$. 